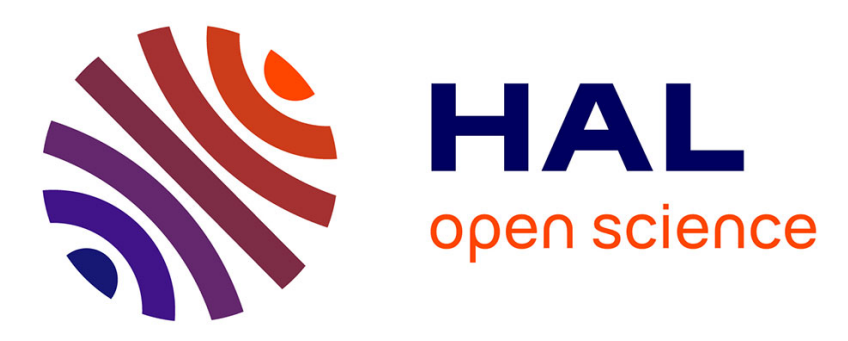

\title{
Scattering theory and cancellation of gravity-flexural waves of floating plates
}

\author{
M. Farhat, P.-Y. Chen, H. Bagci, K. N Salama, A. Alù, S. Guenneau
}

\section{To cite this version:}

M. Farhat, P.-Y. Chen, H. Bagci, K. N Salama, A. Alù, et al.. Scattering theory and cancellation of gravity-flexural waves of floating plates. Physical Review B, 2020, 101 (1), 10.1103/PhysRevB.101.014307 . hal-03139952

\section{HAL Id: hal-03139952 https://hal-amu.archives-ouvertes.fr/hal-03139952}

Submitted on 12 Feb 2021

HAL is a multi-disciplinary open access archive for the deposit and dissemination of scientific research documents, whether they are published or not. The documents may come from teaching and research institutions in France or abroad, or from public or private research centers.
L'archive ouverte pluridisciplinaire HAL, est destinée au dépôt et à la diffusion de documents scientifiques de niveau recherche, publiés ou non, émanant des établissements d'enseignement et de recherche français ou étrangers, des laboratoires publics ou privés. 


\title{
Scattering theory and cancellation of gravity-flexural waves of floating plates
}

\author{
M. Farhat, ${ }^{1,}{ }^{*}$ P.-Y. Chen, ${ }^{2}$ H. Bagci, ${ }^{1}$ K. N. Salama,,${ }^{1}$ A. Alù, ${ }^{3}$ and S. Guenneau ${ }^{4}$ \\ ${ }^{1}$ Division of Computer, Electrical, and Mathematical Science and Engineering, \\ King Abdullah University of Science and Technology (KAUST), Thuwal 23955-6900, Saudi Arabia \\ ${ }^{2}$ Department of Electrical and Computer Engineering, University of Illinois at Chicago, Chicago, Illinois 60607, USA \\ ${ }^{3}$ Photonics Initiative, Advanced Science Research Center, City University of New York, New York 10031, USA \\ ${ }^{4}$ Aix Marseille Univ, CNRS, Centrale Marseille, Institut Fresnel, Marseille, France
}

(Received 1 May 2019; revised manuscript received 21 December 2019; published 28 January 2020; corrected 10 July 2020)

\begin{abstract}
We combine theories of scattering for linearized water waves and flexural waves in thin elastic plates to characterize and achieve control of water wave scattering using floating plates. This requires manipulating a sixth-order partial differential equation with appropriate boundary conditions of the velocity potential. Making use of multipole expansions, we reduce the scattering problem to a linear algebraic system. The response of a floating plate in the quasistatic limit simplifies, considering a distinct behavior for water and flexural waves. Unlike for similar studies in electromagnetics and acoustics, scattering of gravity-flexural waves results in a nonvanishing scattering cross-section in the zero-frequency limit, dominated by its zeroth-order multipole. Potential applications lie in floating structures manipulating ocean water waves.
\end{abstract}

DOI: 10.1103/PhysRevB.101.014307

\section{INTRODUCTION}

In recent years, there has been a growing interest in studying the scattering of various kinds of waves from random and composite media [1,2]. Scattering cancellation technique (SCT) is an active topic of research directly related to scattering analysis, and it relies on covering objects with shells of opposite dipole moment to cancel their scattering response in the quasistatic limit [3-5]. This technique has been generalized to account for various types of waves [2]. In the same vein, the practical importance of designing offshore floating structures and buildings, such as airports or oil plants, triggered the interest in characterizing water wave propagation and its effects on these structures [6-18]. These structures can be modeled as thin elastic plates (TEPs), and their interaction with water waves obeys a modified version of the biharmonic equation [19]. In this paper, we make use of the scattering theory to rigorously characterize this interaction. Moreover, the present study is motivated by earlier work on manipulation of water waves via lensing [20] and cloaking [2] with structures clamped to the waterbed, which may be transposed to floating structures [21-23].

In this contribution, an equation with sixth-order derivatives and its appropriate boundary conditions are used to model the scattering and propagation of linearized water waves, off TEPs. This equation is derived from the combined Euler and plate theories [24-28] and used in characterizing scattering from an object floating on shallow water [29] as schematized in Fig. 1(a). We then analyze the scattering response of the floating elastic structure shown in Fig. 1(b), which consists of a cylindrical disc in a TEP, in the presence of a water wave excitation (time-harmonic vibration of the liquid free surface in the vertical $z$ direction). It is assumed that the

*mohamed.farhat@kaust.edu.sa out-of-plane dimension [the $x z$ plane in Fig. 1(a)] of the floating plate is negligible compared to its in-plane dimensions [the $x y$ plane in Fig. 1(a)] [24]. We show that in the quasistatic limit, i.e., for $\beta_{1} r_{1} \ll 1$, where $\beta_{1}$ is the gravity-flexural wave number and $r_{1}$ is the radius of the scatterer, the scattering is dominated by the zeroth-order multipole term unlike for electrodynamics where the first significant order is the dipolar one. This is not the only marked difference between the two scenarios: the sixth-order gravity-flexural partial differential equation (PDE), which typically describes the propagation of bending waves in TEP floating atop incompressible fluids, is not equivalent to the vector/scalar wave equations that describe electromagnetic or acoustic wave propagation. Consequently, in view of Ref. [30], one can anticipate new types of Mie resonant modes and different wave physics.

\section{FORMULATION OF THE PROBLEM}

In the case of isotropic and uniform physical parameters, the equation governing gravity-flexural waves (in terms of the velocity potential) can be simplified to (See Eqs. (S1)-(S9) in the Supplemental Material [31])

$$
\Delta^{3} \xi_{1}+\beta_{1}^{6} \xi_{1}=0
$$

where $\Delta$ is the Laplacian operator and $\beta_{1}=\left(\rho \omega^{2} / h D\right)^{1 / 6}$ is the gravity-flexural wave number, with $\rho$ the density of the fluid (taken to be $1029 \mathrm{~kg} / \mathrm{m}^{3}$ ), $h$ its depth (taken to be $20 \mathrm{~m}$, except if otherwise stated) $\xi_{1}$ represents the velocity potential (See Supplemental Material [31]). It should be understood that the operator is defined in the two-dimensional space (thin-plate approximation) of the free water surface. The wave number of the water wave satisfies the dispersion relation $\omega^{2}=g k_{0} \tanh \left(h k_{0}\right)$. The dispersion relations for $\beta_{1}$ and $k_{0}$ are depicted in Fig. S1 [31] along with that for the flexural wave number $\beta=\left(M \omega^{2} / D\right)^{1 / 4}$, where $M$ is the mass density of the plate. Similarly, for the water waves, one obtains in the 

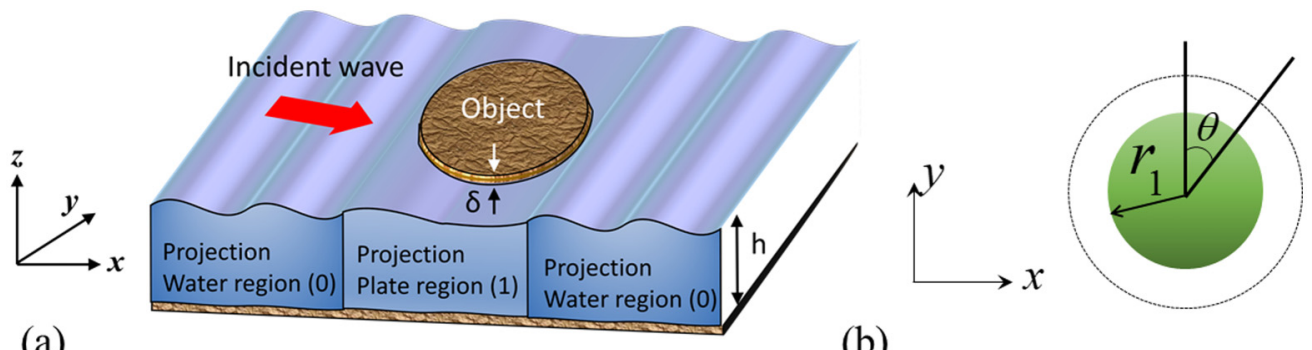

(a)

(b)

FIG. 1. (a) A thin cylindrical elastic plate of thickness $\delta$ floating atop water of depth $h$ and regions of the scattering problem. The water region is denoted by 0 and the plate region by 1 . (b) Top view of a cylindrical thin scatterer to be considered in the first part of this study.

frequency domain

$$
\Delta \xi_{0}+k_{0}^{2} \xi_{0}=0
$$

for the velocity potential in region 0 . Equation (1) is supplemented with six boundary conditions, in the case of a plateplate interface, namely, $\xi_{1}, \partial_{r} \xi_{1}, \Delta \xi_{1}, \partial_{r}\left(\Delta \xi_{1}\right), M_{r}\left(\Delta \xi_{1}\right)$, and $V_{r}\left(\Delta \xi_{1}\right)$ (see the Supplemental Material [31]). An object of radius $r_{1}$ is located atop an incompressible liquid. For $r \leqslant r_{1}$, the wave number is $\beta_{1}$. For $r>r_{1}$, the wave number is $k_{0}$. The object is a TEP that can be made of lightweight concrete, made with low-density aggregate (to make it floating) that has Young modulus $E_{1}=25 \times 10^{9} \mathrm{~Pa}$, Poisson's ratio $v_{1}=0.2$, and density of $800 \mathrm{~kg} / \mathrm{m}^{3}$ [32]. NB that the density of the plate does not intervene in Eq. (1) nor in the dispersion relation and boundary conditions; only the density of the fluid has an effect, through the dispersion relation of gravity-flexural waves. We assume that the object is illuminated by a water plane wave propagating in the $x$-direction, so that $k x=k_{0} r \cos \theta$. The water wave velocity potential due to the incident plane wave is thus expressed as $\xi^{\mathrm{inc}}=e^{i k_{0} r \cos \theta}$, equivalently it can be expanded as $\xi^{\text {inc }}(r, \theta)=\sum_{n=0}^{\infty} \varepsilon_{n} i^{n} J_{n}\left(k_{0} r\right) \cos n \theta$, where the coefficients $\varepsilon_{0}=1$ and $\varepsilon_{n}=2, n \geqslant 1$. At this point a rewriting of Eq. (1) is mandatory to expand the remaining displacement fields in terms of Bessel and Hankel functions. In fact, the velocity potentials $\xi(r, \theta)$ must be finite at $r=0$ and satisfy the radiation condition at $r \rightarrow \infty$. Thus, Eq. (1) is recast as

$$
\Delta^{3} \xi_{1}+\beta_{1}^{6} \xi_{1}=\left(\Delta+\beta_{1}^{2}\right)\left(\Delta-\beta_{1}^{2} \alpha\right)\left(\Delta-\beta_{1}^{2} \alpha^{*}\right) \xi_{1}=0,
$$

where $\alpha=1 / 2(1+i \sqrt{3})$ and the superscript star denotes the complex conjugate here and in the sequel. Hence $\xi_{1}$ is a superposition of solutions to the Helmholtz equation with real (first) and complex (second and third terms) conjugate gravity-flexural wave numbers $\beta_{\alpha}=\beta_{1} \sqrt{\alpha}$ and $\beta_{\alpha}^{*}=\beta_{1} \sqrt{\alpha^{*}}$ with $\sqrt{\alpha}=1 / 2(\sqrt{3}+i)$. Thus, the first term in Eq. (3) results in expansion in terms of $J_{n}\left(\beta_{1} r\right)$. The second and third terms result in expansions in terms of $I_{n}\left(\beta_{\alpha} r\right)$ and $I_{n}\left(\beta_{\alpha}^{*} r\right)$, respectively, the modified Bessel functions of order $n$. Using all these assumptions, the field inside the object is

$$
\begin{aligned}
\xi_{1}(r, \theta)= & \sum_{n=0}^{\infty} \varepsilon_{n} i^{n}\left[B_{n} J_{n}\left(\beta_{1} r\right)+C_{n} I_{n}\left(\beta_{\alpha} r\right)\right. \\
& \left.+E_{n} I_{n}\left(\beta_{\alpha}^{*} r\right)\right] \cos n \theta, \quad r<r_{1},
\end{aligned}
$$

whereas the scattered velocity potential field in region 0 , by taking into account Eq. (2) is

$$
\xi_{0}^{\mathrm{sca}}(r, \theta)=\sum_{n=0}^{\infty} \varepsilon_{n} i^{n}\left[A_{n} H_{n}^{(1)}\left(k_{0} r\right)\right] \cos n \theta, \quad r>r_{1} .
$$

Here, $H_{n}^{(1)}(),. J_{n}($.$) , and I_{n}($.$) are cylindrical Hankel functions$ of the first kind, Bessel and modified Bessel functions, respectively. To solve for the coefficients in the above equations, continuity relations (detailed in the Supplemental Material [31]) are used at the boundary at $r=r_{1}$, for each azimuthal order $n$. This yields a matrix system of equations in unknown coefficients $A_{n}, B_{n}, C_{n}$, and $E_{n}$. The far-field scattering amplitude $f(\theta)=\sqrt{2 r} e^{-i\left(k_{0} r-\pi / 4\right)} \lim _{r \rightarrow \infty} \xi_{0}^{\text {sca }}(r, \theta)$ is a measure of the object's visibility in direction $\theta$ and has the unit of a square root of length [26]. The total scattering cross-section (SCS) is $\sigma^{\text {sca }}=1 / 2 \int_{0}^{2 \pi} d \theta|f(\theta)|^{2}$. It may thus be expressed as

$$
\sigma^{\text {sca }}=\frac{4}{k_{0}} \sum_{n=0}^{\infty} \varepsilon_{n}\left|A_{n}\right|^{2} .
$$

The unknown coefficients in Eqs. (4) and (5) satisfy the linear system

$$
\left(\begin{array}{cccc}
-H_{n}^{(1)}\left(k_{0} r_{1}\right) & J_{n}\left(\beta_{1} r_{1}\right) & I_{n}\left(\beta_{\alpha} r_{1}\right) & I_{n}\left(\beta_{\alpha}^{*} r_{1}\right) \\
-k_{0} H_{n}^{(1)^{\prime}}\left(k_{0} r_{1}\right) & \beta_{1} J_{n}^{\prime}\left(\beta_{1} r_{1}\right) & \beta_{\alpha} I_{n}^{\prime}\left(\beta_{\alpha} r_{1}\right) & \beta_{\alpha}^{*} I_{n}^{\prime}\left(\beta_{\alpha}^{*} r_{1}\right) \\
0 & V_{J_{n}}\left(\beta_{1} r_{1}\right) & V_{I_{n}}\left(\beta_{\alpha} r_{1}\right) & V_{I_{n}}\left(\beta_{\alpha}^{*} r_{1}\right) \\
0 & W_{J_{n}}\left(\beta_{1} r_{1}\right) & W_{I_{n}}\left(\beta_{\alpha} r_{1}\right) & W_{I_{n}}\left(\beta_{\alpha}^{*} r_{1}\right)
\end{array}\right)\left[\begin{array}{c}
A_{n} \\
B_{n} \\
C_{n} \\
E_{n}
\end{array}\right]=\left[\begin{array}{c}
J_{n}\left(k_{0} r_{1}\right) \\
k_{0} J_{n}^{\prime}\left(k_{0} r_{1}\right) \\
0 \\
0
\end{array}\right]
$$

with the prime denoting the first derivative of the special functions, and the expressions of the functionals $V_{Z_{n}}$ and $W_{Z_{n}}$ given in the Supplemental Material [31]. The expression of $A_{n}$ can be obtained from Eq. (7) by using the Cramer's rule
[24], i.e., $A_{n}=\operatorname{det}\left(M_{n, 1}\right) / \operatorname{det}\left(M_{n}\right)$, where $M_{n}$ is the matrix in the LHS of Eq. (7) and the matrix $M_{n, 1}$ is obtained from $M_{n}$ by interchanging $H_{n}^{(1)}$ and $J_{n}$ (see the Supplemental Material [31] for more details). If we consider objects satisfying the 


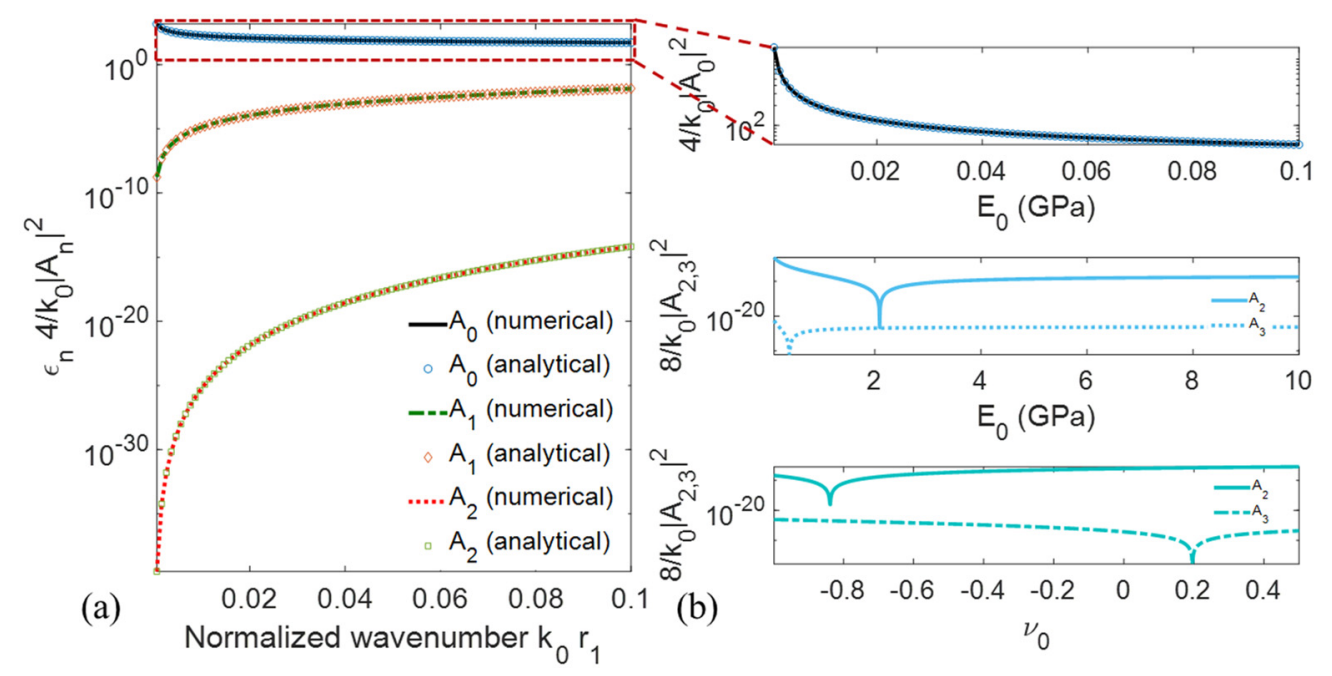

FIG. 2. (a) Normalized scattering coefficients (for $n=0,1$, and 2) versus the normalized wave number obtained using Eqs. (8) and (9) and Eqs. (S15) and (S16) plotted using markers, and numerically using Eq. (7) plotted using lines. (b) Upper panel: Zoom of the dominant scattering order $4 / k_{0}\left|A_{0}\right|^{2}$. Middle and lower panels give the dependence of $8 / k_{0}\left|A_{2}\right|^{2}$ and $8 / k_{0}\left|A_{3}\right|^{2}$ versus the Young modulus and Poisson's coefficient of the TEP.

quasistatic condition $k_{0} r_{1} \ll 1$ and $\beta_{1, \alpha} r_{1} \ll 1$, then only the first few of the scattering terms contribute to the scattering cross-section.

\section{ANALYTICS AND NUMERICS}

In fact, solving Eq. (7) for $n=0,1,2,3$ under the quasistatic condition yields the coefficients

$$
\begin{gathered}
A_{0}=\frac{2 i \pi}{1-4 \gamma_{e}+2 i \pi+\log (16)-4 \log \left(k_{0} r_{1}\right)}+o\left(\left(k_{0} r_{1}\right)^{2 / 3}\right), \\
A_{1}=-i \frac{3 \pi}{16}\left(k_{0} r_{1}\right)^{2}+o\left(\left(k_{0} r_{1}\right)^{2}\right) .
\end{gathered}
$$

The expressions of $A_{2}$ and $A_{3}$ are given in the Supplemental Material [31]. It can be seen that the dominant orders $A_{0}$ and $A_{1}$ depend only on the radius of the object. Also, one has $A_{n \geqslant 2}=O\left(\left(k_{0} r_{1}\right)^{2(n+1)}\right)$. Additionally, the observation that the contribution of $A_{0}$ to the SCS is infinite at zero frequency is intriguing, since it means that $\sigma^{\text {sca }}$ diverges at zero-frequency, which is counterintuitive. The only example that possesses somehow similar features is that of pinned holes in TEP, in the context of flexural waves (obeying the biharmonic equation), as was analyzed in Refs. [26,33,28,34]. However, in that case, the scattering object was described only by its radius, i.e., there was no flexural field inside it, and the object had no other physical parameters, except its geometrical dimension. By contrast, here, the object is a TEP, floating atop of water, and there is a field inside it, as can be seen from Eq. (4). The object has also physical parameters (density, Young modulus, Poisson's ratio, etc.). And still, the dominant scattering order $A_{0}$ is independent of all these properties, except for the radius $r_{1}$. This observation is of tremendous importance to the last section of this paper, where SCT is considered for such class of waves. It is also reminiscent of zero-frequency bandgaps $[33,34]$. Figure 2(a) gives the plot of these coefficients versus the normalized wave number which are compared against the numerical calculations using Eq. (7).

The dependence of $A_{n}, n=2,3$ on the Young modulus and the Poisson ratio of the TEP for $k_{0} r_{1}=0.1$ is given in Fig. 2(b) middle and lower panels, respectively. These have dips (zeros) and they occur for example for $A_{2}$ when $r_{1}^{4}\left(\rho_{0} g / D_{1}\right)\left(303-v_{1}\left(v_{1}-18\right)\right)=-\iota_{1}\left(v_{1}-1\right)\left(v_{1}+3\right) \iota_{1}=$ 3840 (See Supplemental Material [31]). The expressions given in Eqs. (8) and (9) and Eqs. (S15) and (S16) are only valid for small arguments. So to characterize the scattering from the objects shown in Fig. 1, one needs to numerically solve the algebraic system of Eq. (7) and compute the different scattering coefficients, and ultimately the SCS, and verify the convergence with respect to $N$, that is the number of coefficients used in Eq. (6). Then, by verifying the convergence of the SCS, we plot it versus the normalized wave number in Fig. 3(a), where it can be seen that multiple Mie resonances occur across the considered spectral region. This plot is given for a moderate value of the thickness of the plate, i.e., $\delta=1 \mathrm{~m}$, compared with the water wave wavelength.

In this case for small wave numbers, the quasistatic limit applies and the scattering coefficients follow Eqs. (8) and (9) and Eqs. (S15) and (S16). However, for very small thickness on the order of a few centimeters, this limit does not apply anymore, since $k_{0} r_{1} \ll 1$ but $\beta_{1} r_{1}>1$. This change of regime is shown in the inset of Fig. 3(b). The SCS is given for this scenario in Figs. 3(b) and 3(c), where the latter is a magnified view of the former. These plots show a completely different behavior, whereby one observes scattering maxima, for small wave numbers, and these scattering resonances are dominant, compared to the classical higher frequency Mie resonance [35]. In Fig. 3(c) we can see that these resonances are ultra-narrowband and of Fano line-shape. We further note that the smaller the thickness of the plate, the higher the zero-frequency scattering, as can be clearly seen in Figs. 3(b) and 3(c).

The angular dependence of the SCS is depicted in Fig. 3(d), where we plot it versus the angle of observation for three 

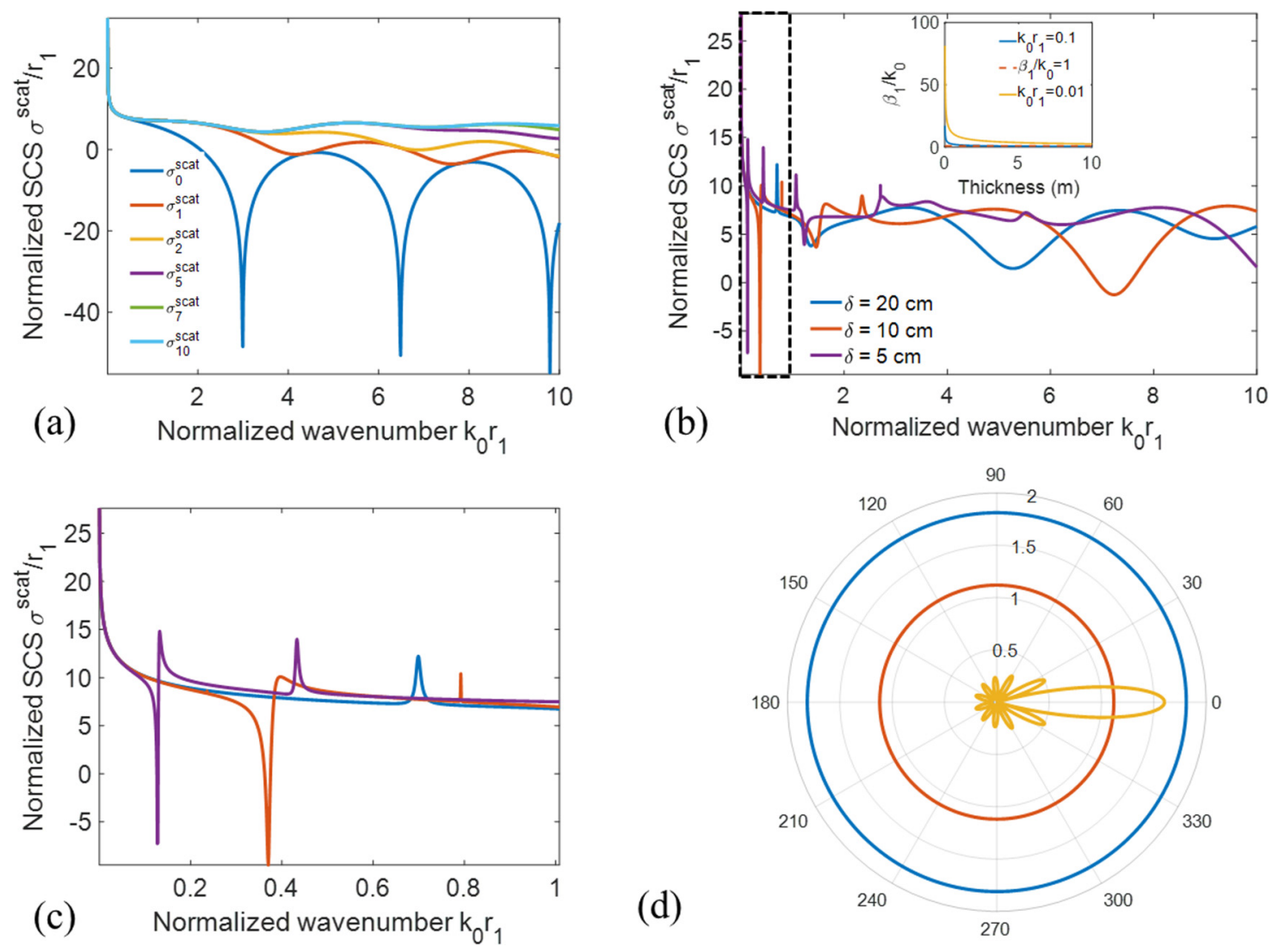

(d)

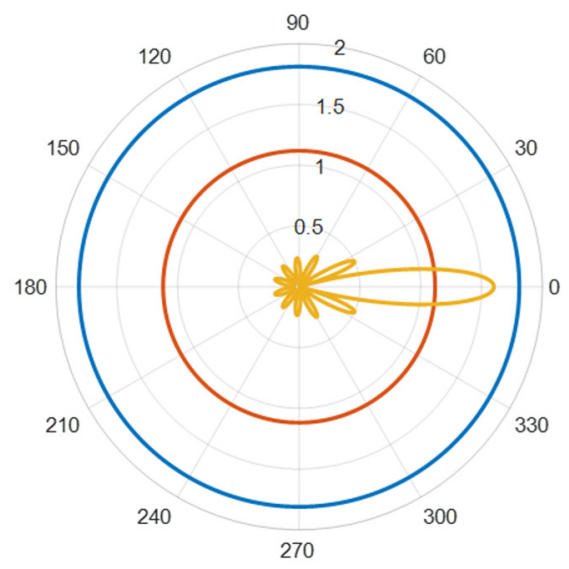

FIG. 3. (a) SCS of the cylindrical TEP (with $\delta=1 \mathrm{~m}, v_{1}=0.2$, and $E_{1}=25 \mathrm{GPa}$ ), with varying number of multipole orders (e.g., $\sigma_{5}^{\text {scat }}$ is the SCS with 5 scattering orders), until convergence is reached. (b) Total SCS (converged) for the same cylindrical TEP, but with a thickness of the plate of $0.2 \mathrm{~m}, 0.1 \mathrm{~m}$, and $0.05 \mathrm{~m}$. The inset shows the regime change of the gravity-flexural wave number versus the thickness of the plate for different wave numbers of the water waves. (c) Magnified view of (b) in the low-frequency regime. (d) Angular dependence of the scattering cross-section of the same object as in (a) for $k_{0} r_{1}=0.1$ (blue), $k_{0} r_{1}=1$ (orange), and $k_{0} r_{1}=10$ (yellow).

representative wave numbers. Enhanced and isotropic Mie resonance can be seen, quite remarkably for the smaller wave numbers, in contrast to usual scattering scenarios. For the larger wave numbers, the amplitude of the scattering is reduced and becomes strongly anisotropic. This features is unique to this kind of scattering. It should be mentioned too that for very small thickness [red curve in Fig. 3(c)] for some modes, the maximum is preceded by a minimum, where the SCS goes to zero, which is again reminiscent of Fano resonances. This also shows that the cylindrical plate becomes nearly invisible for some frequencies without implementation of a cloaking system.

\section{ON CLOAKING AND SCATTERING CANCELLATION OF GRAVITY-FLEXURAL WAVES}

Let us now consider cloaking the object as can be seen in the inset of Fig. 4(a). An object (made of lightweight concrete) of radius $r_{1}$ with thickness $5 \mathrm{~m}$ is thus covered with a shell of outer radius $r_{2}$, and both float atop water. Two scenarios can be considered at this point: (i) The core-shell is immediately surrounded by water. (ii) The core-shell lies at the center of a homogeneous TEP floating atop water.

Scenario (i): For $r \leqslant r_{1}$, the gravity-flexural wave number is $\beta_{1}$. For $r_{1}<r \leqslant r_{2}$, the gravity-flexural wave number is $\beta_{2}$. And for $r>r_{2}$, the water wave number is $k_{0}$, as before. The object and the shell are TEP that have the flexural rigidity $D_{1,2}$, the Young modulus $E_{1,2}$, and the Poisson's ratio $v_{1,2}$, respectively. The field inside region 1 is given by

$$
\begin{aligned}
\xi_{1}(r, \theta)= & \sum_{n=0}^{\infty} \varepsilon_{n} i^{n}\left[B_{n} J_{n}\left(\beta_{1} r\right)+C_{n} I_{n}\left(\beta_{\alpha, 1} r\right)\right. \\
& \left.+E_{n} I_{n}\left(\beta_{\alpha, 1}^{*} r\right)\right] \cos n \theta, \quad r<r_{1} .
\end{aligned}
$$

In region 2, i.e., inside the cloaking shell, one has

$$
\begin{aligned}
\xi_{2}(r, \theta)= & \sum_{n=0}^{\infty} \varepsilon_{n} i^{n}\left[F_{n} Y_{n}\left(\beta_{2} r\right)+G_{n} K_{n}\left(\beta_{\alpha, 1} r\right)\right. \\
& +L_{n} K_{n}\left(\beta_{\alpha, 2}^{*} r\right)+N_{n} J_{n}\left(\beta_{2} r\right)+O_{n} I_{n}\left(\beta_{\alpha, 2} r\right) \\
& \left.+P_{n} I_{n}\left(\beta_{\alpha, 2}^{*} r\right)\right] \cos n \theta, \quad r_{1}<r \leqslant r_{2},
\end{aligned}
$$

whereas the scattered velocity potential field (i.e., in region 0 ) is similar to Eq. (5). To solve for the 10 unknown coefficients in the above equations, continuity relations (all details are given in the Supplemental Material [31]) are used at both boundaries. If we consider objects satisfying $k_{0} r_{1,2} \ll 1$ and $\beta_{\alpha, 1} r_{1,2} \ll 1$, then only the first few $A_{n}$ coefficients contribute to the SCS. In fact, solving Eq. (S17) for $n=0,1,2$, under 


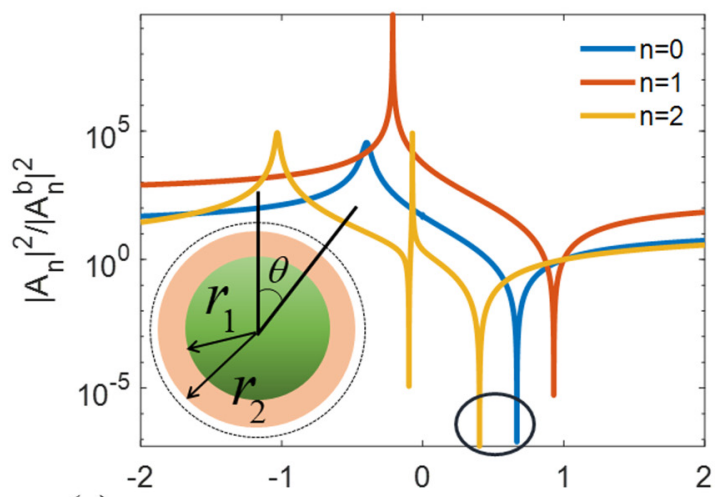

(a) Normalized Young modulus $\mathrm{E}_{2} / \mathrm{E}_{0}$

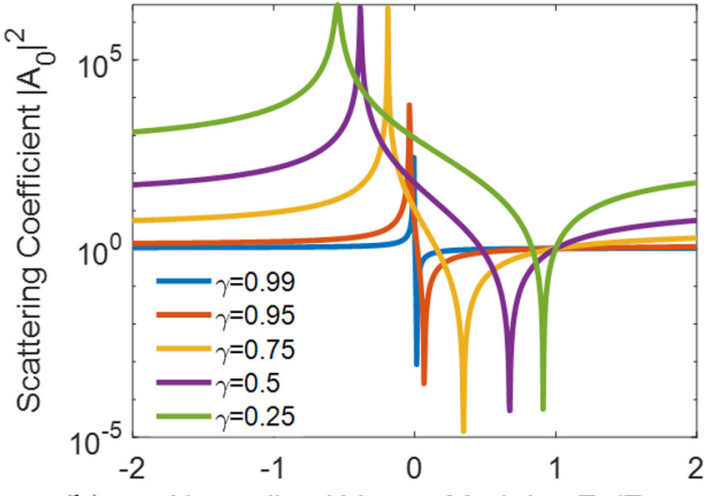

(b) Normalized Young Modulus $\mathrm{E}_{2} / \mathrm{E}_{0}$

$$
10 \log _{10}\left(\sigma_{\mathrm{c}}^{\mathrm{sca}} / \sigma_{\mathrm{b}}^{\mathrm{sca}}\right)
$$

(c)
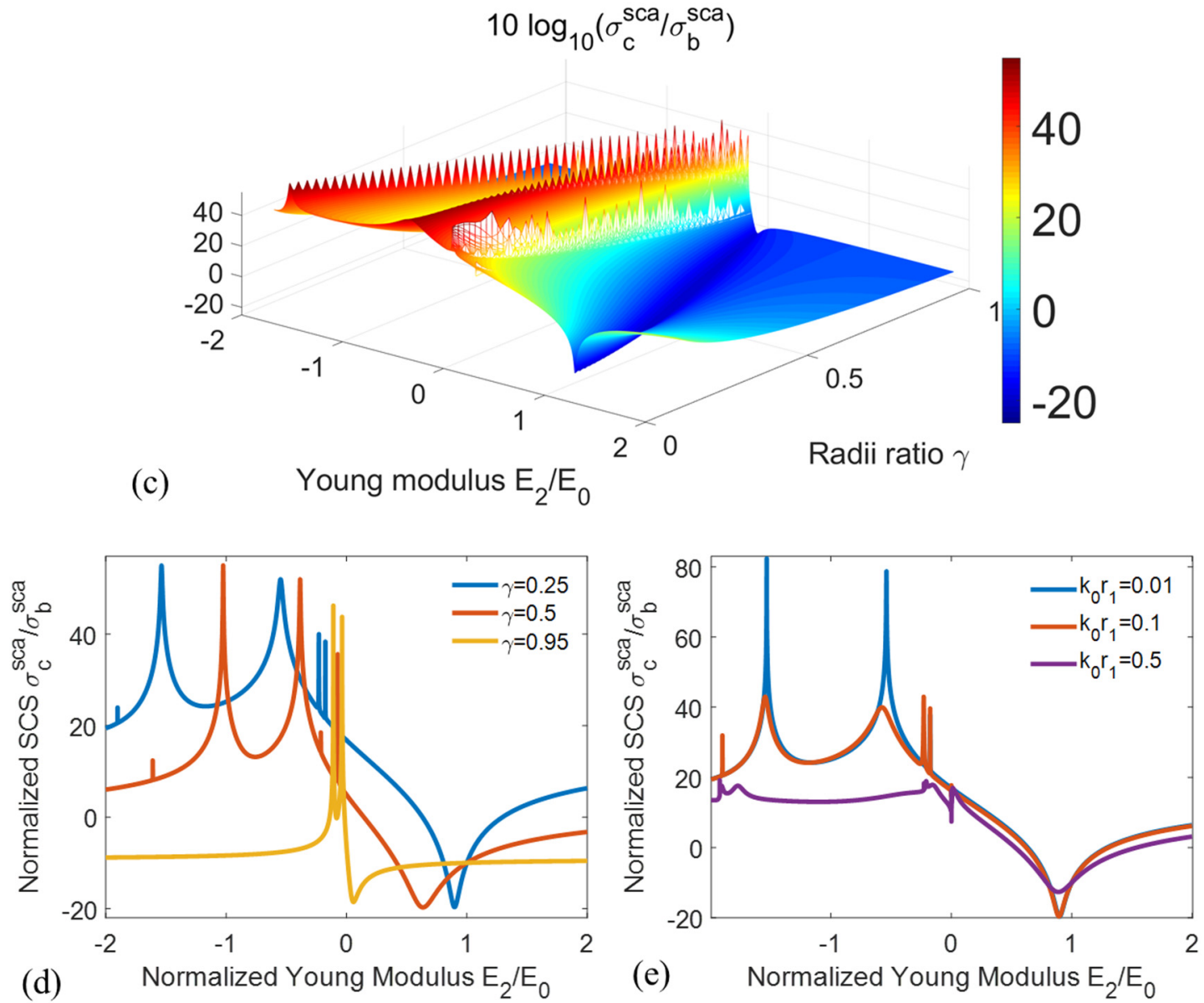

FIG. 4. (a) Normalized scattering coefficients vs. the Young modulus of the core-shell structure, for $k_{0} r_{1}=0.15$ and $\gamma=r_{1} / r_{2}=0.5$. The inset gives the geometry of the core-shell structure. (b) Dominant scattering order for various radii of the cloaking structure. (c) Contour plot of the SCS versus the Young modulus and radius of the cloak. The plot is in logarithmic scale [as shown in the title of Fig. 4(c)] and normalized to the bare object SCS. (d) Normalized SCS of the same structure for different radii and a fixed wave number $\left(k_{0} r_{1}=0.05\right)$ and (e) same for different wave numbers and a fixed radius $(\gamma=0.25)$.

the quasistatic condition yields

$A_{0}=\frac{2 i \pi}{1-4 \gamma_{e}+\log (16)-4 \log \left(k_{0} r_{2}\right)+2 i \pi}+o\left(\left(k_{0} r_{1}\right)^{2 / 3}\right)$,

where $\gamma_{e}$ is the Euler-Mascheroni constant. Since $k_{0} r_{2} \ll 1$, the expression of Eq. (12) is a constant. Thus, it can be seen that irrespective of the physical parameters of the coating shell, $A_{0}$ cannot be made equal to zero, and thus SCT in this context is not possible. Moreover, if we normalize it with respect to the wave number, one can see that the SCS becomes singular, i.e.,

$$
\sigma^{\text {sca }} \approx \frac{4}{k_{0}}\left|A_{0}\right|^{2} \approx \frac{\pi^{2}}{\left(k_{0} \log \left(k_{0} r_{2}\right)\right)^{2}} .
$$

And owing to the fact that $\lim _{x \rightarrow 0}(x \log x)=0, \sigma^{\text {sca }}$ diverges in the zero-frequency limit. This scenario of singular 
scattering is reminiscent of the case of clamped biharmonic (purely flexural) obstacles [26]. An analogous scenario occurs for microwaves of certain polarization in the case of a thin metal wire, and this has been used to dilute the average concentration of electrons and considerably enhance the effective electron mass through self-inductance [36]. It is interesting to note also that the dominant order scattering $A_{0}$ is independent of the parameters of the inner object, including its radius. This is interesting for potential applications in control of long wavelength ocean waves as this indicates some zero frequency stop-bands could be achieved for water waves propagating through an array of periodically distributed floating objects [34]. The analogous scenario occurring for flexural (Lamb) waves propagating within periodically clamped plates has led to the design of an earthquake shield [34]. We further note that zero frequency photonic stopbands associated with periodically distributed infinite conducting wires [37] are associated with noncommuting limits in homogenization theory.

Scenario (ii): Let us move now to the second case, described earlier. The derivation of the scattering orders is done in a similar way (all details can be found in the Supplemental Material [31]). The main difference with scenario (i) is that here the dominant scattering orders are nonsingular.

The homogeneous TEP has a Young modulus $E_{0}=25 \mathrm{GPa}$ and the depth of water is a before assumed to be $20 \mathrm{~m}$. The object to cloak has a Young modulus $E_{1}=10 \times E_{0}$ and a Poisson ratio $v_{1}=2 \times v_{0}=0.5$. Hence, $A_{0}$ and $A_{2}$ (dominant coefficients) are shown to be of the same order (see Fig. S2 in the Supplemental Material [31]). In Fig. 4(a) the first three coefficients (normalized by the bare coefficients) are given versus the normalized Young modulus of the cloak (the other physical parameters of the cloak are taken to be equal to those of the free-space TEP). This plot shows the possibility, in particular, to separately cancel $A_{0}$ and $A_{2}$ but for different values of $E_{2}$. Figure 4(b) shows the dependence of $A_{0}$ on the Young modulus but for different radii of the cloak.

The main message from this type of cloaking that was not observed for other types of waves is that to perform SCT one needs to cancel two different scattering orders. This happens at different physical parameters of the cloak. Indeed, this means that optimization may be necessary to drastically cancel the total SCS in the quasistatic limit. The main result is thus shown in Fig. 4(c) where the SCS is normalized versus the SCS of the bare object and plotted against the Young modulus and radius of the cloak.
For some physical values, a drastic scattering cancellation takes place (dark blue color). Also for other parameters a huge scattering can be observed (dark red color), as typically observed in the SCT, and that may be explained by a Fano-like behavior of the waves. To clearly show this effect, the SCS is plotted against $E_{2} / E_{0}$ for few values of $\gamma$ for $k_{0} r_{1}=0.05$ in Fig. 4(d) and for few values of $k_{0} r_{1}$ for $\gamma=0.25$ in Fig. 4(e). The same results can be obtained for a thicker object, i.e., in the range of $10 \mathrm{~m}$, but for longer wavelengths (see the Supplemental Material [31] for further characterization of this type of cloaking).

\section{CONCLUSIONS}

We analyzed in detail the scattering of gravity-flexural waves propagating when a TEP lies atop the water surface. Scattering from a single cylindrical object was first investigated and low-frequency Mie resonances were discovered. Additionally, by coating the cylindrical object, SCT was shown to be impossible to realize, irrespective of the physical parameters of the shell, which is a paradigm shift compared with SCT for other types of waves. This unusual behavior can be understood, since the incident wave is different from the waves that propagate inside the plate. In a second scenario, we considered a core-shell structure with a gravity-flexural wave incident on it (is instead considered and), which was shown to possess cloaking features. Motivated by the search for zero frequency stop-band structures in different wave systems, such as the recently achieved seismic shields in sedimentary soils structured by arrays of clamped columns to a bedrock [38], we would like to now build upon the present work to analyze Floquet-Bloch waves propagating within a doubly periodic array of floating plates. We believe that our work opens unprecedented avenues in the control of water waves making use of floating metamaterial structures.

\section{ACKNOWLEDGMENT}

The authors thank anonymous referees for their useful comments that helped improve the presentation of this work. S.G. wishes to thank the Department of Mathematics at Imperial College London for a visiting position in the group of Prof. R.V. Craster in 2018-2019 (funded by EP-SRC grant EP/L024926/1).
[1] L. B. Felsen and N. Marcuvitz, Radiation and Scattering of Waves (IEEE Press, Piscataway, NJ, 1994).

[2] M. Farhat, P.-Y. Chen, S. Guenneau, and S. Enoch, Transformation Wave Physics: Electromagnetics, Elastodynamics, and Thermodynamics (CRC Press, Boca Raton, FL, 2016).

[3] A. Alù and N. Engheta, Phys. Rev. E 72, 016623 (2005).

[4] A. Alù and N. Engheta, Opt. Express 15, 3318 (2007).

[5] B. Luk'yanchuk, R. Paniagua-Domínguez, A. I. Kuznetsov, A. E. Miroshnichenko, and Y. S. Kivshar, Philos. Trans. R. Soc. A 375, 20160069 (2017).
[6] G. Zilman and T. Miloh, Appl. Ocean Res. 22, 191 (2000).

[7] G. Rehbinder and P. Yakubenko, J. Geophys. Res. 104, 10827 (1999).

[8] T. Williams and V. Squire, J. Fluid Mech. 569, 113 (2006).

[9] L. Bennetts and T. Williams, Proc. R. Soc. A 471, 20140698 (2015).

[10] R. Porter, Wave Motion 66, 118 (2016).

[11] R. Porter, J. Fluids Struct. 84, 171 (2019).

[12] E. Skelton, R. Craster, A. Colombi, and D. Colquitt, New J. Phys. 20, 053017 (2018).

[13] H. Lamb, Proc. R. Soc. A 98, 205 (1920). 
[14] N. Balmforth and R. Craster, J. Fluid Mech. 395, 89 (1999).

[15] J. J. Stoker, Water Waves: The Mathematical Theory with Applications (John Wiley \& Sons, New York, 2011), Vol. 36.

[16] D. V. Evans and T. V. Davies, Wave-Ice Interaction (Springer, Berlin, 1968).

[17] M. H. Meylan, J. Fluid Mech. 454, 387 (2002).

[18] M. A. Peter, M. H. Meylan, and H. Chung, Int. J. Offshore Polar Eng. 14, 81 (2004).

[19] E. Rizzuto and C. G. Soares, Sustainable Maritime Transportation and Exploitation of Sea Resources (CRC Press, Boca Raton, FL, 2011).

[20] M. Farhat, S. Guenneau, S. Enoch, and A. Movchan, J. Comput. Appl. Math. 234, 2011 (2010).

[21] M. Kashiwagi, T. Iida, and M. Miki, in Proceedings of the 30th International Workshop on Water Waves and Floating Bodies (Bristol, UK, 2015).

[22] R. Read, H. B. Bingham, and J. Newman, in Proceedings of the 31st International Workshop on Water Waves and Floating Bodies (Plymouth, MI, USA, 2016).

[23] A. Zareei and R. Alam, in Proceedings of the 31 st International Workshop on Water Waves and Floating Bodies (Plymouth, MI, USA, 2016), p. 197.

[24] S. P. Timoshenko and S. Woinowsky-Krieger, Theory of Plates and Shells (McGraw-Hill, New York, 1959).

[25] K. F. Graff, Wave Motion in Elastic Solids (Courier Corporation, North Chelmsford, MA, 2012).

[26] A. Norris and C. Vemula, J. Sound Vib. 181, 115 (1995).
[27] N. Stenger, M. Wilhelm, and M. Wegener, Phys. Rev. Lett. 108, 014301 (2012).

[28] M. Farhat, P.-Y. Chen, H. Bağcı, S. Enoch, S. Guenneau, and A. Alu, Sci. Rep. 4, 4644 (2014).

[29] E. J. Kubatko, S. Bunya, C. Dawson, J. J. Westerink, and C. Mirabito, J. Sci. Comput. 40, 315 (2009).

[30] M. Farhat, P.-Y. Chen, S. Guenneau, K. N. Salama, and H. Bağc1, Phys. Rev. B 95, 174201 (2017).

[31] See Supplemental Material at http://link.aps.org/supplemental/ 10.1103/PhysRevB.101.014307 for supplementary figures and equations.

[32] T. Kh. Mohammad Ali, J. Mat. Eng. Structures 5, 57 (2018).

[33] A. Movchan, N. Movchan, and R. McPhedran, Proc. R. Soc. A 463, 2505 (2007).

[34] G. Lefebvre, T. Antonakakis, Y. Achaoui, R. Craster, S. Guenneau, and P. Sebbah, Phys. Rev. Lett. 118, 254302 (2017).

[35] B. Stout and R. McPhedran, Europhys. Lett. 119, 44002 (2017).

[36] J. B. Pendry, A. Holden, W. Stewart, and I. Youngs, Phys. Rev. Lett. 76, 4773 (1996).

[37] N. Nicorovici, R. McPhedran, and L. Botten, Phys. Rev. Lett. 75, 1507 (1995).

[38] Y. Achaoui, T. Antonakakis, S. Brule, R. Craster, S. Enoch, and S. Guenneau, New J. Phys. 19, 063022 (2017).

Correction: Incorrect values were given for density below Eq. (2) and for the Young modulus of the homogeneous TEP given in Sec. IV and have been fixed. 